\title{
Clinical manifestations of homozygote allele carriers in Huntington disease
}

Esther Cubo, MD, PhD, Saul-Indra Martinez-Horta, PhD, Frederic Sampedro Santalo, Asunción Martínez Descalls, PhD, Sara Calvo, PhD, Cecilia Gil-Polo, MD, PhD, Ignacio Muñoz, MD, Katia Llano, Natividad Mariscal, Dolores Diaz, Aranzazu Gutierrez, Laura Aguado, and María A. Ramos-Arroyo, MD, PhD, for the European HD Network

Neurology ${ }^{\circledR}$ 2019;92:e2101-e2108. doi:10.1212/WNL.0000000000007147

Abstract

\section{Objective}

Because patients homozygous for Huntington disease (HD) receive the gain-of-function mutation in a double dose, one would expect a more toxic effect in homozygotes than in heterozygotes. Our aim was to investigate the phenotypic differences between homozygotes with both alleles $\geq 36$ CAG repeats and heterozygotes with 1 allele $\geq 36$ CAG repeats.

\section{Methods}

This was an international, longitudinal, case-control study (European Huntington's Disease Network Registry database). Baseline and longitudinal total functional capacity, motor, cognitive, and behavioral scores of the Unified Huntington's Disease Rating Scale (UHDRS) were compared between homozygotes and heterozygotes. Four-year follow-up data were analyzed using longitudinal mixed-effects models. To estimate the association of age at onset with the length of the shorter and larger allele in homozygotes and heterozygotes, regression analysis was applied.

\section{Results}

Of 10,921 participants with $\mathrm{HD}$ (5,777 female [52.9\%] and 5,138 male [47.0\%]) with a mean age of $55.1 \pm 14.1$ years, 28 homozygotes $(0.3 \%)$ and $10,893(99.7 \%)$ heterozygotes were identified. After correcting for multiple comparisons, homozygotes and heterozygotes had similar age at onset and UHDRS scores and disease progression. In the multivariate linear regression analysis, the longer allele was the most contributing factor to decreased age at $\mathrm{HD}$ onset in the homozygotes $(p<0.0001)$ and heterozygotes $(p<0.0001)$.

\section{Conclusions}

CAG repeat expansion on both alleles of the HTT gene is infrequent. Age at onset, HD phenotype, and disease progression do not significantly differ between homozygotes and heterozygotes, indicating similar effect on the mutant protein.

\section{Classification of evidence}

This study provides Class II evidence that age at onset, the motor phenotype and rate of motor decline, and symptoms and signs progression is similar in homozygotes compared to heterozygotes.

\author{
Correspondence \\ Dr. Cubo \\ mcubo@ \\ saludcastillayleon.es
}

\section{MORE ONLINE}

$\rightarrow$ Class of Evidence

Criteria for rating therapeutic and diagnostic studies

NPub.org/coe

From the Neurology Department (E.C., C.G.-P., I.M., K.L., N.M., D.D., A.G., L.A.) and Research Unit (S.C.), Hospital Universitario Burgos; Movement Disorders Unit, Neurology Department (S.-I.M.-H., F.S.S.), Hospital de La Santa Creu I Sant Pau, Barcelona; Centro de Investigación en Red-Enfermedades Neurodegenerativas (CIBERNED) (S.-I.M.-H., F.S.S.), Madrid; Neurology Department (A.M.D.), Fundación Jiménez Diez, Madrid; and Genetic Department (M.A.R.-A.), Complejo Hospitalario de Navarra, Pamplona, Spain. 


\section{Glossary}

BMI = body mass index; $\mathbf{C I}=$ confidence interval; EHDN = European Huntington's Disease Network; HD = Huntington disease; SNP = single nucleotide polymorphism; TFC = total functional capacity; TMS = total motor score; UHDRS = Unified Huntington's Disease Rating Scale.

\section{Huntington disease progression: Homozygotes vs heterozygotes

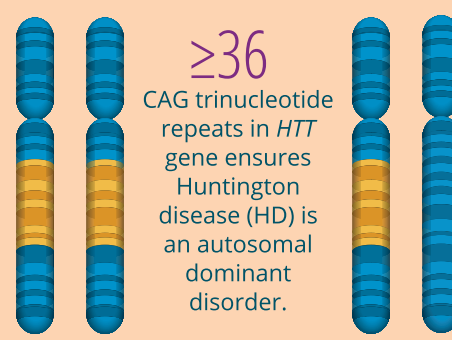 \\ Patients homozygous for HD receive gain-of-function mutation via double dose vs heterozygotes.

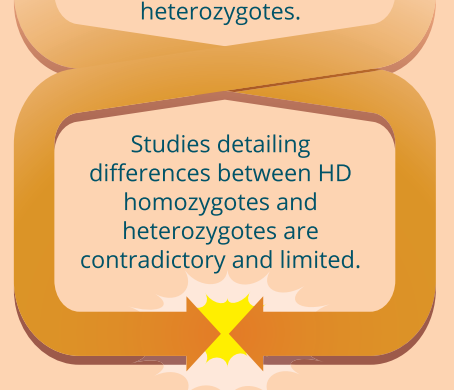 \\ NPub.org/929901 \\ Copyright (c) 2019 American Academy of Neurology}

The presence of 36 or more CAG trinucleotide repeats in the HTT gene nearly ensures the development of Huntington disease (HD) as an autosomal dominantly transmitted disorder. Because homozygote patients (i.e., with 2 mutant alleles) for $\mathrm{HD}$ receive the gain-of-function mutation in a double dose, one would expect a more toxic effect in homozygotes than in the heterozygotes (i.e., with one mutant allele), similar to other poly CAG diseases. ${ }^{1,2}$ In this regard, some publications in animal models and small HD human retrospective studies have reported a more aggressive $\mathrm{HD}$ progression and brain atrophy in homozygotes. ${ }^{2,3}$ In contrast, other authors have reported an indistinguishable phenotype in HD homozygotes and heterozygotes. ${ }^{4-8}$ Given these contradictory data and the sparse longitudinal information, we aimed to further investigate the clinical differences between HD homozygotes (with both alleles $\geq 36$ CAG repeats) and heterozygotes (with one allele $\geq 36$ CAG repeats) in terms of age at onset, phenotypic presentation, and disease progression.

\section{Methods}

\section{Design}

This was an international, retrospective-longitudinal, casecontrol study.

\section{Sample characteristics and ethics}

Clinical and sociodemographic data were obtained from patients enrolled in the European Huntington's Disease Registry Database (European Huntington's Disease Network $[\mathrm{EHDN}]) .{ }^{9}$ For this observational study, participants provided written, informed consent following the International Conference on Harmonization-Good Clinical Practice guidelines. ${ }^{10}$ For participants who lacked capacity to consent, study sites followed country-specific guidelines for signing consent forms. Minors agreed with both parents authorizing for them. Ethical approval was collected from the local ethics committee for each study site contributing to EHDN Registry. ${ }^{9}$

\section{Participants and clinical assessments}

Data of individuals from the European Huntington's Disease Registry Database, from July 1998 to December 2016, with a larger allele $\geq 36$ CAG repeats within the Huntingtin gene were included in this study. Data collection adhered to a standard protocol including electronic case report forms, and used identical study protocols of assessment and sampling of biomaterials.

Demographics, number of years of education, and body mass index (BMI, calculated as weight in kilograms divided by 
height in meters squared) were extracted from the EHDN registry. Motor and psychiatric signs were scored using the Unified Huntington's Disease Rating Scale (UHDRS). ${ }^{11}$ For motor and behavior UHDRS, higher scores indicated worse motor and higher psychiatric impairment. For cognition, we used the cognitive UHDRS composite score (UHDRS total correct for letter fluency, Symbol Digit Modalities Test, and Stroop subscores for word reading, color identification, naming, and interference), with lower scores indicating worse performance. ${ }^{12}$ Disease stage was obtained from total functional capacity (TFC) scores, with higher scores indicating better functional status. ${ }^{13}$ Patients were followed up on a yearly basis according to the EHDN Registry protocol. ${ }^{9,12}$ Study site raters were annually trained, evaluated, and certified to lessen interrater and intrarater variability. Data entry was reviewed online and an on-site by monitors fluent in the language of the study site. ${ }^{9,12}$ The HTT CAG genotyping was performed at each local genetic laboratory. In addition, fresh blood samples were donated by patients and sent to the central laboratory in Milan, Bio-Rep, to be reanalyzed. Clinically significant discrepancies, defined as crossing the boundary at 35-36 or 39-40 CAG repeat lengths, or measurement errors ( \pm 1 for CAG repeat lengths $\leq 42$ and \pm 3 for CAG repeat lengths $\geq 43$ ), were brought to the attention of the local site investigator and subsequently addressed.

\section{Data management}

HD homozygotes were defined as carriers of 2 alleles with $\geq 36$ CAG repeats, while HD heterozygotes were individuals with the longer allele $\geq 36 \mathrm{CAG}$ repeats and the shorter allele $<36$ CAG repeats. Demographics, CAG repeat length, and clinical information including total motor score (TMS), cognitive and behavior UHDRS scores, and TFC information at baseline and after 4 years of follow-up were collected from the EHDN database. With the motor UHDRS, different domain subscores were calculated: chorea (sum of the chorea items [face, buccolingual, upper and lower extremities scores]), dystonia (sum of the trunk, upper and lower extremities scores), bradykinesia (sum of the finger taps, pronate/ supinate, rigidity of each extremity, and body bradykinesia scores), gait impairment (sum of gait, tandem, and retropulsion scores), and oculomotor performance (sum of ocular pursuit, saccade initiation, and saccade velocity). To deal with missing values, case-wise deletions were adopted.

\section{Statistical analysis}

Analysis was done using IBM-SPSS 21 software (SPSS Inc., Chicago, IL), following the Reporting of Observational Studies in Epidemiology guidelines. ${ }^{14}$ Normal distribution of variables was analyzed using the Kolmogorov-Smirnov test. Descriptive analysis of the participants' characteristics was performed in terms of frequencies (percentage), mean/ median values with the corresponding SD or interquartile range, as appropriate, and $95 \%$ confidence intervals (CI).

Clinical characteristics were analyzed in a cross-sectional and longitudinal manner. Baseline differences between HD homozygotes and the total sample of HD heterozygous were first evaluated. To balance differences in sample sizes, a post hoc secondary analysis was carried out, comparing homozygotes with a subset of heterozygotes, paired by age and CAG larger allele (1:3). In addition, because aging might worsen the UHDRS scores, especially bradykinesia and gait scores, independently of the genetic status, ${ }^{15}$ a comparative analysis between homozygotes and heterozygotes was conducted including young and older participants. All homozygote and heterozygote participants were classified as older ( $>51$ years old) or younger participants ( $\leq 51$ years old), based on the median age of homozygotes at registry entry. Differences were analyzed using the $\chi^{2}, \mathrm{Phi}$, and Cramer tests (categorical variables), and Mann-Whitney $U$ tests (nonparametric for quantitative variables). A significance level of $\alpha$ $=0.004,2$-sided tests, was applied after post hoc Bonferroni multiple comparison adjustments.

To analyze the association between age, length of the larger and shorter alleles, BMI, and UHDRS outcome measures, correlations were calculated using Spearman (nonparametric) correlation coefficients. To analyze the relationship between age at onset and the length of the larger and shorter alleles in the homozygote and heterozygote groups, a multivariate linear regression analysis was conducted.

For follow-up data, linear mixed-effects models ${ }^{16}$ were constructed to investigate the course of different outcomes over a 4-year period. To account for the correlation between repeated measurements on the same participant, a random intercept and random time effect (slope) per participant was used. For all analyses, an unstructured covariance for the random intercepts and random slopes was used. Differences in the rate of progression (i.e., slope) between homozygotes and heterozygotes were compared.

\section{Data availability statement}

We used the same methodology as that described in a previous study. ${ }^{15}$ The European HD Registry is a large, prospective study observing the natural course, clinical spectrum, and management of HD in 140 centers from 17 European countries and 3 other countries. ${ }^{9,12}$ More information on the Registry can be found at euro-hd.net/html/ registry. This study is registered with ClinicalTrials.gov, number NCT01590589.

\section{Results}

As of December 2016, of 10,921 participants with $\mathrm{HD}(5,777$ female [52.9\%] and 5,138 male [47.0\%]) with a mean age of $55.1 \pm 14.1$ years, 28 homozygotes $(0.3 \%)$ and 10,893 (99.7\%) heterozygotes were identified. The median CAG repeat lengths of the longer and shorter alleles were 45 (42; $47)$ and $38(37 ; 40)$, respectively, for the homozygote group, and $43(44 ; 45)$ and $18(17 ; 20)$, respectively, for the heterozygote group (table 1 ). 
Table 1 Baseline characteristics of homozygotes and heterozygotes with Huntington disease (HD)

\begin{tabular}{|c|c|c|c|c|c|}
\hline & Homozygotes, $n=28$ & Heterozygotes, $n=10,893$ & $p$ Value $^{a}$ & Heterozygotes $_{p}, \mathrm{n}=65$ & $p$ Value $^{b}$ \\
\hline Age, y & 51.5 (36.7-71.7), 28 & $55.0(45.0-65.0), 10,886$ & 0.31 & $52.0(39.5-71.5), 65$ & 0.76 \\
\hline Female & 19 (67.9), 28 & $5,758(52.9), 10,893$ & 0.12 & $38(58.5), 65$ & 0.48 \\
\hline Shorter allele, CAG repeats & 38 (37-40), 28 & $18(17-20), 10,893$ & $<0.0001$ & $18(17-21), 65$ & $<0.0001$ \\
\hline Longer allele, CAG repeats & 45 (42-47), 28 & 43 (41-46), 10,893 & 0.03 & 45 (41-47), 65 & 0.37 \\
\hline Age at onset, y & $39.5(23.7-53.0), 20$ & $46.0(37.0-56.0), 8,442$ & 0.04 & $44.5(34.7-58.0), 46$ & 0.13 \\
\hline HD duration, y & $12.5(6.0-16.0), 20$ & $10.0(6.0-15.0), 8,478$ & 0.35 & $12.0(9.0-16.2), 46$ & 0.98 \\
\hline Education, y & 10.0 (9.0-13.0), 27 & $11.0(9.0-14.0), 10,391$ & 0.12 & $12.0(10.0-14.0), 65$ & 0.01 \\
\hline BMI & $22.1(21.0-23.6), 25$ & 23.5 (21.2-26.5), 10,817 & 0.04 & $23.2(20.9-26.7), 59$ & 0.13 \\
\hline TFC & $9.5(4.5-12.7), 28$ & $10.0(6.0-13.0), 10,717$ & 0.50 & $11.0(7.0-13.0), 65$ & 0.15 \\
\hline TMS-UHDRS & 35.0 (8.2-57.7), 28 & $27.0(9.0-46.0), 10,445$ & 0.46 & $21.5(4.0-45.0), 64$ & 0.20 \\
\hline Chorea-UHDRS & $6.5(0.2-10.7), 28$ & $5.0(1.0-10.0), 10,576$ & 0.55 & $4.0(0.0-9.0), 65$ & 0.33 \\
\hline Bradykinesia-UHDRS & $8.0(3.2-16.7), 28$ & $7.0(2.0-12.0), 10,535$ & 0.44 & $6.0(1.0-11.0), 65$ & 0.28 \\
\hline Dystonia-UHDRS & $1.0(0.0-5.7), 28$ & $0.0(0.0-3.0), 10,562$ & 0.35 & $1.0(0.0-2.0), 65$ & 0.12 \\
\hline Gait-UHDRS & $4.0(0.0-6.7), 28$ & $3.0(0.0-5.0), 10,542$ & 0.23 & $1.5(0.0-4.7), 65$ & 0.15 \\
\hline Ocular-UHDRS & $6.0(1.5-16.5), 28$ & $6.0(1.2-11.0), 10,569$ & 0.31 & $4.0(0.0-10.0), 65$ & 0.06 \\
\hline Cognitive-UHDRS & $226.0(142.0-269.0), 9$ & 174.0 (122.0-241.0), 4,102 & 0.34 & 210.0 (127.0-302.0), 35 & 0.71 \\
\hline Behavior-UHDRS & $13.0(4.0-27.0), 21$ & $12.0(5.0-21.0), 6,753$ & 0.81 & $8.0(3.0-15.0), 47$ & 0.51 \\
\hline
\end{tabular}

Abbreviations: $\mathrm{BMI}=$ body mass index; Heterozygotes $=$ age-larger allele-paired heterozygotes; TFC = total functional capacity; TMS = total motor score; UHDRS = Unified Huntington's Disease Rating Scale.

A significance level of $a=0.004$, 2-sided tests was applied after post hoc Bonferroni multiple comparisons adjustments. Values are expressed as median (interquartile range), $\mathrm{n}$; or $\mathrm{n}(\%), \mathrm{N}$.

${ }^{a} p$ Value relates to the homozygotes vs heterozygotes comparison.

${ }^{\mathrm{b}} p$ Value relates to homozygotes vs age-larger allele-paired heterozygotes. Baseline comparison values were compared using the Mann-Whitney $U$ test.

At baseline, clinical characteristics of homozygotes and heterozygotes were not significantly different, although homozygotes showed a trend for lower age at $\mathrm{HD}$ onset and lower BMI (table 1 and figure 1). Similar results were obtained in the post hoc comparative analysis between homozygotes and the age-larger allele-paired heterozygotes, with a trend for greater ocular disturbances in homozygotes (table 1).

After stratification of participants by age, the median CAG repeats of the longer allele in the younger group was $47(45 ; 55)$ among homozygotes $(\mathrm{n}=14)$ and $45(43 ; 49)$ among heterozygotes $(n=4,138)$, while in the older group, it was $43(40 ; 45)$ and $42(41 ; 44)$ for homozygotes $(n=14)$ and heterozygotes $(n$ $=6,757$ ), respectively. No significant differences in BMI, TMS, Cognitive, Behavior-UHDRS, and TFC scores were observed in the young or old group, except for a trend for lower age at $\mathrm{HD}$ onset in young homozygotes compared to young heterozygotes $(27.0[16.7 ; 36.5]$ vs $34.0[28.0 ; 39.0], p=0.04)$ and greater gait UHDRS scores in old homozygotes compared to old heterozygotes $(5.0[4.0 ; 9.2]$ vs $4.0[2.2 ; 7.0], p=0.01)$.

The length of the longer allele was correlated with age at $\mathrm{HD}$ onset in both homozygotes $\left(r_{\mathrm{s}}=-0.86, p<0.0001\right)$ and heterozygotes $\left(r_{s}=-0.76, p<0.0001\right)$, while correlation with BMI was only seen in homozygotes $\left(r_{\mathrm{s}}=-0.50, p=0.01\right)$. The length of the shorter allele did not have a significant effect on cognitive, behavior, or motor (including bradykinesia, chorea, gait, and dystonia UHDRS) scores, in either group. Likewise, the number of years of education did not show a significant correlation with age at $\mathrm{HD}$ onset among heterozygotes or homozygotes.

In the multivariate linear regression analysis, using the smaller and longer alleles as the independent variables, the length of the longer allele contributed most to earlier age at $\mathrm{HD}$ onset in the homozygote and heterozygote groups (table 2). According to these models, in heterozygotes, with 1-unit CAG repeat increase in the longer allele, age at $\mathrm{HD}$ onset decreased 1.90 years, 95\% CI $-1.94 ;-1.86(p<0.0001)$. Likewise, in homozygotes, for 1-unit CAG repeat increase in the longer allele, age at HD onset decreased 1.72 years, 95\% CI $-2.36 ;-1.07(p<0.0001)$. These models explained $44.8 \%$ and $61.7 \%$ of the variability of age at $\mathrm{HD}$ onset in the heterozygote and homozygote groups, respectively.

TMS, cognitive, behavior UHDRS, BMI, and TFC follow-up data are shown in boxplots (figure 2). Overall, no significant 
Figure 1 The relationship between expanded alleles CAG repeat length and age at onset of Huntington disease

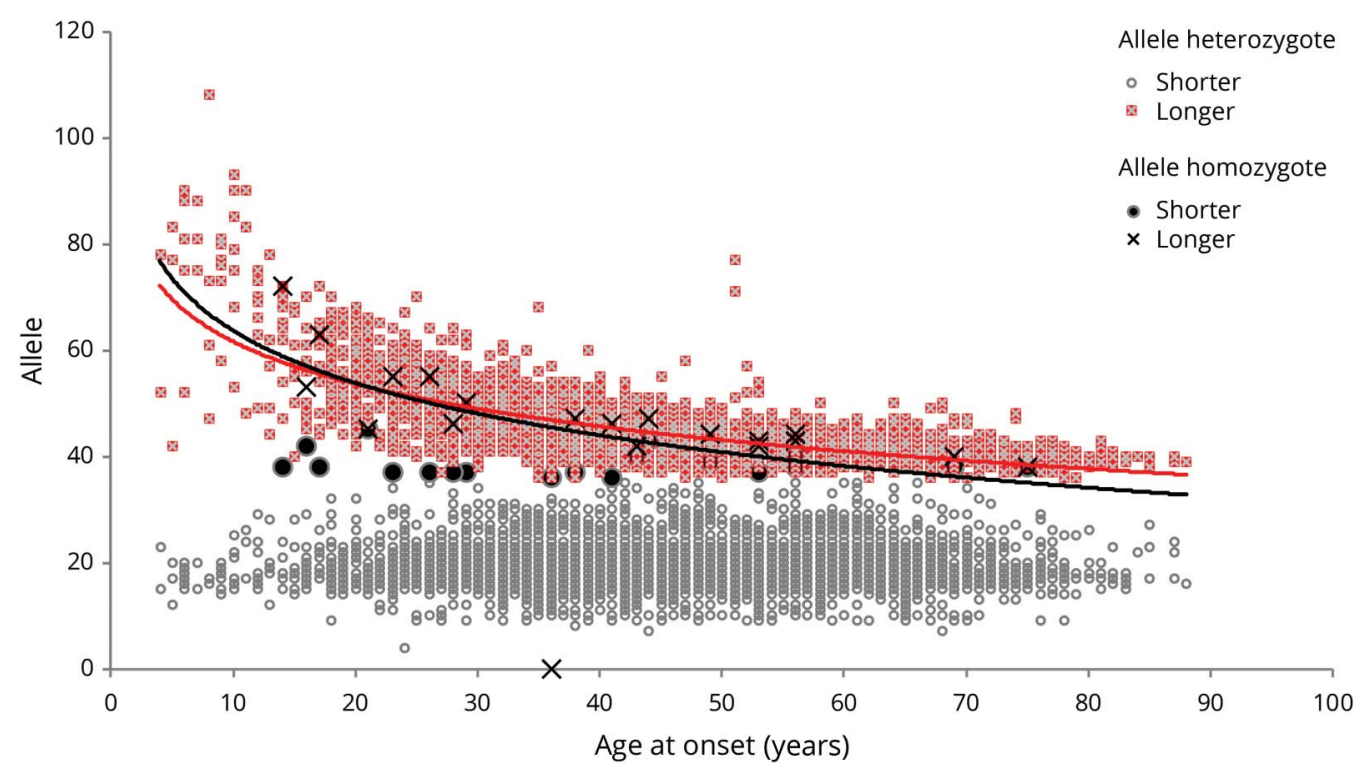

The minimal adequate model for this heterozygote and homozygote dataset is shown as black (homozygotes) and red (heterozygotes) lines.

differences were observed in homozygotes compared to heterozygotes in terms of outcome variables over the course of 4 years (table 3).

\section{Discussion}

In this longitudinal analysis of the EHDN registry, after correcting for multiple comparisons, homozygote $\mathrm{HD}$ carriers had a similar age at onset, phenotype, and disease progression compared to heterozygotes. These findings suggest that, overall, the shorter expanded allele does not have a significant and major influence in determining either the age at onset or the phenotypic expression and progression of $\mathrm{HD}$.

In $\mathrm{HD}$ animal models and some CAG triplet diseases in humans, including spinal and bulbar muscular atrophy and dentatorubral-pallidoluysian atrophy, homozygotes have consistently shown more aggressive neurodegeneration compared to heterozygotes. ${ }^{17,18}$ In other polyQ diseases, however, the effect of a second expanded allele remains unclear. Single reports and small series of homozygotes for spinocerebellar ataxia type 6 and 3 suggest a gene dose effect on age at onset and increased severity of the phenotype. ${ }^{19,20}$ For HD, assessment of double dose gene effect in the phenotype has been extremely challenging, given the rare occurrence of homozygotes. While some publications in $\mathrm{HD}$ have reported an indistinguishable phenotype in homozygotes and heterozygotes, ${ }^{4,7,8}$ other studies have observed a nonchoreiform phenotype presentation, more frequently in homozygotes, with a significant increase in severity of progression of motor and psychiatric manifestations, indicating that homozygotes may present with a wider spectrum of neurologic symptoms, other than chorea, compared to heterozygotes. ${ }^{2,21}$ Supporting findings included marked cerebellum atrophy in neuroimaging in 3 homozygotes and widespread brain atrophy at autopsy of one homozygote patient with HD. ${ }^{2}$ Our study, however, cannot confirm this difference in phenotype, as clinical characteristics of homozygotes were similar to heterozygotes. Even more, we did not observe a different clinical profile between younger and older homozygotes, except for a trend for greater gait impairment in the old group. Homozygotes (especially young homozygotes) had lower age at $\mathrm{HD}$ onset than heterozygotes, but they also had higher median CAG repeats of the longer allele, which may partially account for an earlier initiation of symptoms. Of note, homozygotes also showed a trend for lower BMI compared to

Table 2 Multivariate lineal regression analysis of clinical variables associated with age at Huntington disease onset

\begin{tabular}{|c|c|c|}
\hline & $\begin{array}{l}\text { Homozygotes, corrected } R^{2}=65.7, n=20, n \text { ( } 95 \% \text { confidence } \\
\text { interval), } p \text { value }\end{array}$ & $\begin{array}{l}\text { Heterozygotes, corrected } R^{2}=48.7, \mathrm{n}=8,442, \mathrm{~B} \text { ( } 95 \% \text { confidence } \\
\text { interval), } p \text { value }\end{array}$ \\
\hline $\begin{array}{l}\text { Shorter } \\
\text { allele }\end{array}$ & $-1.44(-3.65$ to 0.76$), 0.18$ & 0.01 ( -0.04 to 0.07$), 0.15$ \\
\hline $\begin{array}{l}\text { Longer } \\
\text { allele }\end{array}$ & $-1.72(-2.36$ to -1.07$),<0.0001$ & $-1.90(-1.94$ to -1.86$),<0.0001$ \\
\hline
\end{tabular}


Figure 2 Follow-up data

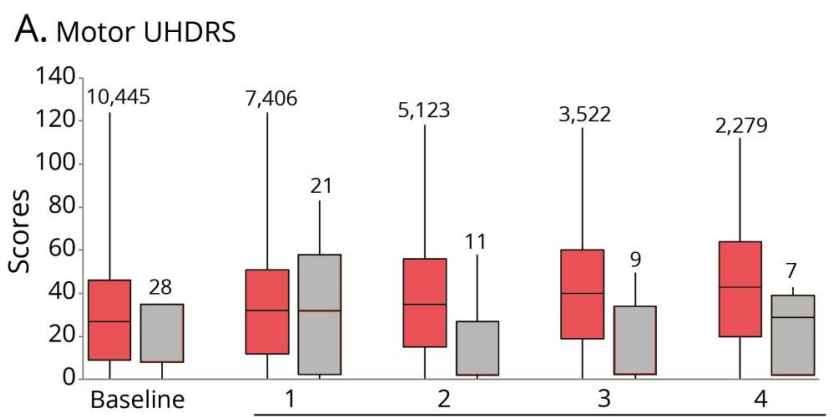

B. Cognitive UHDRS

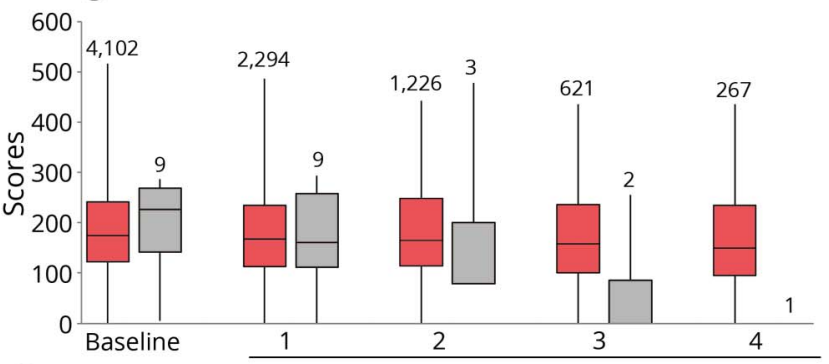

C. Behavior UHDRS

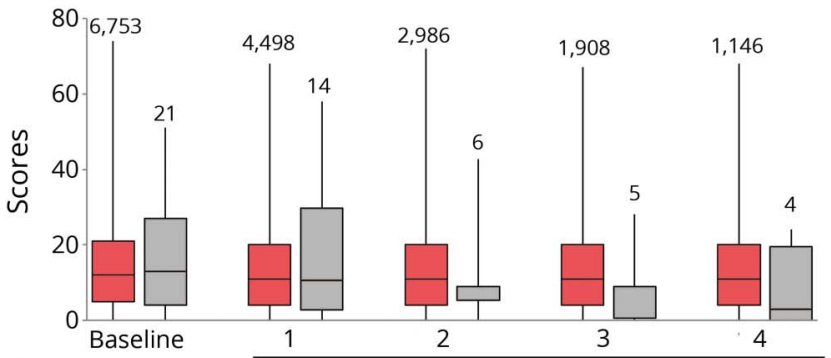

D. Body mass index

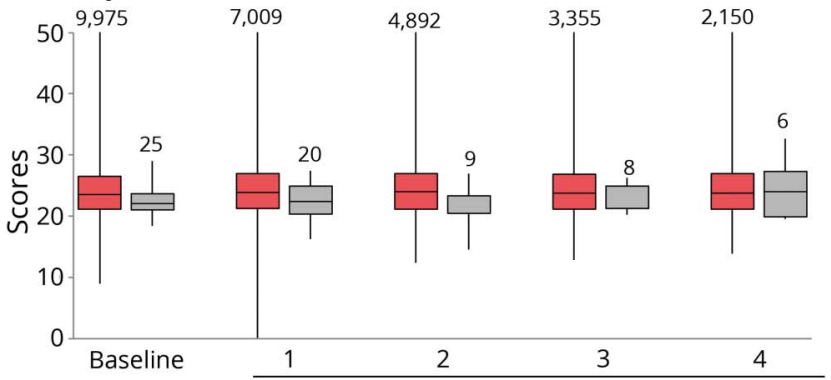

E. Total functional capacity

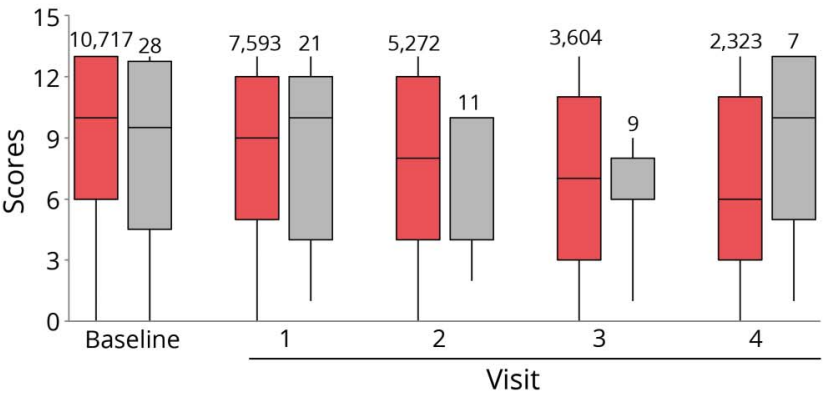

Follow-up data for motor Unified Huntington's Disease Rating Scale (UHDRS) (A), cognitive UHDRS (B), behavior UHDRS (C), body mass index (D), and total functional capacity (E). Number of participants participating in each visit is shown. Homozygotes are represented in gray boxes and heterozygotes in red boxes. The dark line represents the median of the outcome variable, the bottom of the box indicates the 25th percentile, and the top the 75th percentile.
Table 3 Slope differences for the homozygotes vs heterozygotes

\begin{tabular}{lll}
\hline & Slope difference (SE) & $p$ Value \\
\hline TFC & $-0.9(0.6)$ & 0.40 \\
\hline TMS-UHDRS & $4.1(4.2)$ & 0.36 \\
\hline Chorea-UHDRS & $0.2(0.8)$ & 0.78 \\
\hline Bradykinesia-UHDRS & $1.0(1.1)$ & 0.37 \\
\hline Dystonia-UHDRS & $0.5(0.3)$ & 0.44 \\
\hline Ocular-UHDRS & $1.3(1.0)$ & 0.19 \\
\hline Cognitive-UHDRS & $17.0(26.4)$ & 0.53 \\
\hline Behavior-UHDRS & $1.5(2.1)$ & 0.47 \\
\hline BMI & $-1.5(1.2)$ & 0.18 \\
\hline
\end{tabular}

Abbreviations: $\mathrm{BMI}=$ body mass index; TFC = total functional capacity; TMS = total motor score; UHDRS = Unified Huntington's Disease Rating Scale. Shown are parameter estimates from the linear mixed models. The difference between homozygotes and heterozygotes is expressed as a slope. $p$ Values relates to the difference in the homozygotes compared to heterozygotes.

heterozygotes. Explaining hypothesis includes that homozygotes might be at risk for a greater hypermetabolic state that may precede the occurrence of motor symptoms and contribute to weight loss. ${ }^{22,23}$

Heterozygote individuals with clinically diagnosed $\mathrm{HD}$ experience a steady progressive decline in the cardinal features of the disease. ${ }^{24,25}$ In our study, heterozygotes had a similar rate of decline compared to homozygotes in terms of BMI, TFC, TMS, behavior, and cognitive UHDRS scores. In contrast, Squitieri et al. ${ }^{2}$ observed a faster rate of progression in disability, measured by independence and the physical disability scales, in 8 homozygotes compared to 75 heterozygotes. Given the different methodology used in that study, ${ }^{2}$ our results are not comparable. Available clinimetric data show that in the overall HD population, items measured by the TMS-UHDRS, the only recommended rating scale to measure the severity of motor signs in $\mathrm{HD}$ that cover all motor domains in $\mathrm{HD},{ }^{26}$ have variable weights at different $\mathrm{HD}$ stages. It seems that bradykinesia and dystonia are predominant in patients with greater CAG repeats and younger onset of HD compared to lower CAG repeats in the larger allele. Instead, chorea is predominant in earlier stages of manifest, adult $\mathrm{HD}$, tends to plateau, and decreases later, and parkinsonian features become progressively more severe and are more clinically significant in later stages of the disease. ${ }^{27}$ Interestingly, we found a similar motor phenotype progression in homozygotes compared to heterozygotes in terms of choreiform and nonchoreiform manifestations over time, except for a trend for greater gait impairment in older homozygotes compared with heterozygotes in post hoc analysis. However, the scarce and relatively short longitudinal clinical information on homozygotes limits the clinical relevance of these observations. 
In previous studies on $\mathrm{HD}$ homozygotes, the most frequent variable of disease severity was the difference in age at $\mathrm{HD}$ onset. In agreement with other studies, the length of the longer allele was the most contributing and consistent factor associated with age at $\mathrm{HD}$ onset in both homozygotes and heterozygotes. ${ }^{2,4,5,7,8,28}$ These results suggest that CAG repeat expansion in $\mathrm{HD}$ determines age at onset in a fully dominant fashion. ${ }^{28,29}$ Age at HD onset in our homozygote participants was similar to that of the Lee et al. ${ }^{28}$ study, but lower than reported by Squitieri et al. ${ }^{2}$ It should be mentioned, however, that this difference might be due to the greater expansion in the longer allele in our homozygote sample, compared to the Squitieri et al. ${ }^{2}$ study. In any case, the small number of homozygotes in both studies $^{2,28}$ does not allow us to draw definitive conclusions.

The underlying mechanisms of the lack of a significant expression of a double mutant gene dosage in HD are not wellunderstood. On one hand, it has been proposed that biallelic mutation in HD might be deleterious in patients, due to lack of protective function of the wild-type huntingtin or mitochondria impairment. ${ }^{2,30,31}$ On the other hand, the level of mutant huntingtin protein produced from a single allele apparently exceeds any minimum threshold required to trigger pathogenesis (at a rate determined primarily by its CAG repeat length), and that hypothetically neither additional mutant protein nor the absence of any normal protein further alters the rate of pathogenesis leading to motor onset. ${ }^{28}$ In spite of that, it is also important to consider the potential modifying effect of genetic factors on HD clinical course, beside the CAG repeat length of the HTT gene. In a recent genome-wide disease progression association study, single nucleotide polymorphism (SNP) encoding an amino acid change (Pro67Ala) in $\mathrm{MSH}^{32}$ was shown to have an effect on disease progression. Each copy of the minor allele at this SNP was associated with a 0.4 units per year (95\% CI 0.16-0.66) reduction in the rate of change of the TMS-UHDRS, and a reduction of 0.12 units per year (95\% CI 0.06-0.18) in the rate of change of TFC in heterozygotes. ${ }^{32}$ In this scenario, identification and assessment of individual genetic and environmental factors should contribute to define the possible effect of the second expanded allele on the variance of $\mathrm{HD}$ phenotype and progression.

The main limitations of this study are the small sample of homozygotes, the limited clinical relevance of statistically significant results obtained in post hoc analysis, and the lack of biological data including measurement of mutant huntingtin protein levels and other genetic modifiers, neuroimaging, and postmortem pathologic results in the homozygotes supporting our data. Likewise, we cannot rule out that homozygotes have a significant, distinct motor or cognitive phenotype that cannot be adequately captured by the UHDRS, or detected due to sample selection/attribution bias (loss of follow-up in participants with more severe cognitive or motor decline), or limited statistical power. In addition, participants with HD in our study are very likely to be on medication, a factor that could modify the phenotypic expression and outcome measures. Consequently, the HD clinical manifestations and progression in this study may not reflect that of the broader HD population (homozygotes and heterozygotes) who may have less access to such care. However, despite the above limitations, this study documents the clinical profile and disease progression using the largest HD homozygote multicenter sample so far described. Of note, these observational data were obtained from a database without any prespecified hypotheses at the time of data collection, which may preclude sample selection bias.

The results of this study extend previous reports examining the natural history of $\mathrm{HD}$, highlighting the importance of an observational, longitudinal disease registry. Homozygosity in HD does not seem to modify significantly the age at onset, clinical phenotype, or disease progression, except for subtle clinical differences. Environmental factors and compensatory genetic factors might counteract the possible effect of the mutation in a double dose. This speculative hypothesis could be an area for future therapeutic investigation.

\section{Author contributions}

E. Cubo: study concept, design and writing the manuscript. M.A. Ramos-Arroyo: interpretation, critical revision of the manuscript. S.-I. Martinez-Horta: interpretation, critical revision of the manuscript. A. Martinez-Descalls: critical revision of the manuscript. S. Calvo, F. Sampedro Santalo: data management and analysis. C. Gil-Polo, D. Diaz, A. Gutierrez, I. Muñoz, K. Llano, N. Mariscal, L. Aguado: interpretation and critical review of the manuscript.

\section{Acknowledgment}

The authors thank the EHDN Registry Study Group investigators for collecting the data, all participating Registry patients for their time and efforts, and Margaret Kresse for editing the manuscript.

\section{Study funding}

European Huntington Disease Registry (data mining project: \#852).

\section{Disclosure}

E. Cubo has consulting fees for UCB, Allergan, and AbbVie. S. Martinez-Horta, F. Sampedro, A. Martinez-Descals, S. Calvo, C. Gil, I. Muñoz, K. Llano, N. Mariscal, D. Diaz, A. Gutierrez, L. Aguado, and M. Ramos-Arroyo report no disclosures relevant to the manuscript. Go to Neurology.org/ $\mathrm{N}$ for full disclosures.

\section{Publication history}

Received by Neurology July 3, 2018. Accepted in final form January 4, 2019.

\section{References}

1. Gusella J, MacDonald M. No post-genetics era in human disease research. Nat Rev Genet 2002;3:72-79.

2. Squitieri F, Gellera C, Cannella M, et al. Homozygosity for CAG mutation in Huntington disease is associated with a more severe clinical course. Brain 2003;126: 946-955. 
3. Southwell AL, Smith-Dijak A, Kay C, et al. An enhanced Q175 knock-in mouse model of Huntington disease with higher mutant huntingtin levels and accelerated disease phenotypes. Hum Mol Genet 2016;25:3654-3675.

4. Wexler NS, Young AB, Tanzi RE, et al. Homozygotes for Huntington's disease. Nature 1987;326:194-197.

5. Myers RH, Leavitt J, Farrer LA, et al. Homozygote for Huntington disease. Am J Hum Genet 1989;45:615-618.

6. Laccone F, Engel U, Holinski-Feder E, et al. DNA analysis of Huntington's disease: five years of experience in Germany, Austria, and Switzerland. Neurology 1999;53: 801-806.

7. Dürr A, Hahn-Barma V, Brice A, Pêcheux C, Dodé C, Feingold J. Homozygosity in Huntington's disease. J Med Genet 1999;36:172-173.

8. Kremer B, Goldberg P, Andrew SE, et al. A worldwide study of the Huntington's disease mutation: the sensitivity and specificity of measuring CAG repeats. $\mathrm{N}$ Engl J Med 1994;330:1401-1406.

9. Orth M, European Huntington's Disease Network, Handley OJ, et al. Observing Huntington's disease: the European Huntington's disease Network's Registry. J Neurol Neurosurg Psychiatry 2011;82:1409-1412.

10. ICH. Available at: ich.org. Accessed March 1, 2018.

11. Huntington Study Group. Unified Huntington's Disease Rating Scale: reliability and consistency. Mov Disord 1996;11:136-142.

12. Orth M, Handley OJ, Schwenke C, et al. Observing Huntington's disease: the European Huntington's Disease Network's Registry. PLoS Curr 2010;2: RRN1184.

13. Shoulson I. Huntington disease: functional capacities in patients treated with neuroleptic and antidepressant drugs. Neurology 1981;31:1333-1335.

14. Vandenbroucke JP, von Elm E, Altman DG, et al. Strengthening the reporting of observational studies in Epidemiology (STROBE): explanation and elaboration. Int J Surg 2014;12:1500-1524.

15. Cubo E, Ramos-Arroyo MA, Martinez-Horta S, et al. Clinical manifestations of intermediate allele carriers in Huntington disease. Neurology 2016;87:571-578.

16. Verbeke G, Molenberghs G. Linear Mixed Models for Longitudinal Data. New York: Springer; 2000.

17. Sato K, Kashihara K, Okada S, et al. Does homozygosity advance the onset of dentatorubral-pallidoluysian atrophy? Neurology 1995;45:1934-1936.

18. Graham RK, Slow EJ, Deng Y, et al. Levels of mutant huntingtin influence the phenotypic severity of Huntington disease in YAC128 mouse models. Neurobiol Dis 2006;21:444-455.
19. Matsumura R, Futamura N, Fujimoto Y, et al. Spinocerebellar ataxia type 6: molecular and clinical features of 35 Japanese patients including one homozygous for the CAG repeat expansion. Neurology 1997;49:1238-1243.

20. Soga K, Ishikawa K, Furuya $\mathrm{T}$, et al. Gene dosage effect in spinocerebellar ataxia type 6 homozygotes: a clinical and neuropathological study. J Neurol Sci 2017; 373:321-328.

21. Squitieri F, Berardelli A, Nargi E, et al. Atypical movement disorders in the early stages of Huntington's disease: clinical and genetic analysis. Clin Genet 2000;58:50-56.

22. Petersen A, Bjorkqvist M. Hypothalamic-endocrine aspects in Huntington's disease. Eur J Neurosci 2006;24:961-967.

23. Aziz NA, van der Burg JM, Landwehrmeyer GB, et al. Weight loss in Huntington disease increases with higher CAG repeat number. Neurology 2008;71:1506-1513.

24. Dorsey ER, Beck CA, Darwin K, et al. Natural history of Huntington disease. JAMA Neurol 2013;70:1520-1530.

25. Pagan F, Torres-Yaghi Y, Altshuler M. The diagnosis and natural history of Huntington disease. Handb Clin Neurol 2017;144:63-67.

26. Mestre TA, Busse M, Davis AM, et al. Rating scales and performance-based measures for assessment of functional ability in Huntington's disease: critique and recommendations. Mov Disord Clin Pract 2018;5:361-372.

27. Folstein SE, Jensen B, Leigh RJ, Folstein MF. The measurement of abnormal movement: methods developed for Huntington's disease. Neurobehav Toxicol Teratol 1983;5:605-609.

28. Lee JM, Ramos EM, Lee JH, et al. CAG repeat expansion in Huntington disease determines age at onset in a fully dominant fashion. Neurology 2012;78: 690-695.

29. Uhlmann WR, Penaherrera MS, Robinson WP, Milunsky JM, Nicholson JM, Albin RL. Biallelic mutations in Huntington disease: a new case with just one affected parent, review of the literature and terminology. Am J Med Genet A 2015;167A $1152-1160$.

30. Scherzinger E, Sittler A, Schweiger K, et al. Self-assembly of polyglutamine-containing huntingtin fragments into amyloid-like fibrils: implications for Huntington's disease pathology. Proc Natl Acad Sci USA 1999;96:4604-4609.

31. Squitieri F, Cannella M, Sgarbi G, et al. Severe ultrastructural mitochondrial changes in lymphoblasts homozygous for Huntington disease mutation. Mech Ageing Dev 2006; 127:217-220.

32. Hensman Moss DJ, Pardinas AF, Langbehn D, et al. Identification of genetic variants associated with Huntington's disease progression: a genome-wide association study. Lancet Neurol 2017;16:701-711. 


\section{Neurology}

Clinical manifestations of homozygote allele carriers in Huntington disease

Esther Cubo, Saul-Indra Martinez-Horta, Frederic Sampedro Santalo, et al. Neurology 2019;92;e2101-e2108 Published Online before print March 13, 2019

DOI 10.1212/WNL.0000000000007147

This information is current as of March 13, 2019

\section{Updated Information \&} Services

References

Citations

Subspecialty Collections

Permissions \& Licensing

Reprints including high resolution figures, can be found at: http://n.neurology.org/content/92/18/e2101.full

This article cites 30 articles, 9 of which you can access for free at: http://n.neurology.org/content/92/18/e2101.full\#ref-list-1

This article has been cited by 3 HighWire-hosted articles: http://n.neurology.org/content/92/18/e2101.full\#\#otherarticles

This article, along with others on similar topics, appears in the following collection(s):

All CBMRT/Null Hypothesis

http://n.neurology.org/cgi/collection/all_cbmrt_null_hypothesis All Clinical Neurology

http://n.neurology.org/cgi/collection/all_clinical_neurology

All Movement Disorders

http://n.neurology.org/cgi/collection/all_movement_disorders

Chorea

http://n.neurology.org/cgi/collection/chorea

Cohort studies

http://n.neurology.org/cgi/collection/cohort_studies

Huntington's disease

http://n.neurology.org/cgi/collection/huntingtons_disease

Information about reproducing this article in parts (figures,tables) or in its entirety can be found online at:

http://www.neurology.org/about/about_the_journal\#permissions

Information about ordering reprints can be found online:

http://n.neurology.org/subscribers/advertise

Neurology ${ }^{\circledR}$ is the official journal of the American Academy of Neurology. Published continuously since 1951, it is now a weekly with 48 issues per year. Copyright ( 2019 American Academy of Neurology. All rights reserved. Print ISSN: 0028-3878. Online ISSN: 1526-632X.

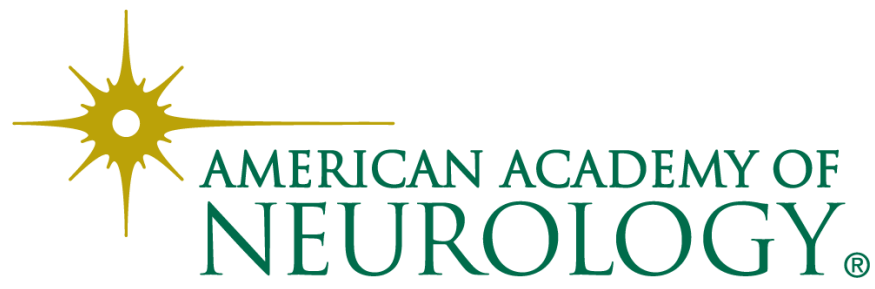

